REVISTA ANDALUZA DE ANTROPOLOGÍA.

NÚMERO 5: APORTACIONES Y POTENCIALIDADES DE LA ANTROPOLOGÍA DE LA SALUD.

SEPTIEMBRE DE 2013

ISSN 2174-6796

[pp. 182-188]

http://dx.doi.org/10.12795/RAA.2013.i05.10

$\square$

\title{
TOLEDO CHÁVARRI, ANA (2012). Antropólogos, caciques e indígenas. Cartografías del desarrollo en el Papaloapan. Málaga: SEPHA, 426 pp.
}

\section{Ignacio Iturralde Blanco \\ Universidad de Barcelona}

Como reconoce la propia autora, el trabajo de campo es un rito de paso. Más aun, si se trata de las primeras inmersiones en él, como nos ocurre a los doctorandos. Más incluso, si se pretendía etnografiar la relación entre un proyecto de desarrollo y los conocimientos locales, pero no queda ni rastro del mismo ${ }^{1}$. Y mucho más, si cabe, si la violencia irrumpe en el proceso de investigación y desencadena un abrupto final homicida que remite al México más bronco. Todo ello no puede menos que producir la consiguiente decepción y la más que justificada pérdida de toda ingenuidad e idealización con la que podías haberte aproximado al objeto de tu investigación. Por tanto, con Antropólogos, caciques e indígenas estamos ante una monografía sobre una región del sur de México, la cuenca del Papaloapan, uno de los sistemas hidrológicos más importantes del país. Un trabajo que reflexiona sobre las poblaciones mazatecas y chinantecas, su relación con la nación y las intervenciones de desarrollo que en sus territorios han producido desde desplazamientos masivos hasta terribles desastres ecológicos (Restrepo, 2009). Pero, al mismo tiempo, estamos ante la etnografía de un descubrimiento y una decepción. Una muy interesante reflexión en torno a la posición investigadora que aboga por la necesidad

1. El títula del proyecto inicial era "Desarrollo local y conocimientos situados" que fue abandonado conforme Ana Toledo Chávarri notó, al llegar al campo en 2006, que el proyecto apenas existía como texto, como narrativa, pero sin ninguna materialización en los pueblos. 
de mantener o recuperar la distancia crítica necesaria para poder abordar ciertos temas, por calmar los movimientos pendulares que algunos descubrimientos producen en nosotros, los antropólogos, por tener la suficiente flexibilidad para abandonar ciertas hipótesis y preconceptos una vez la realidad del campo desborda cuanto podías haber imaginado en los despachos de tu universidad de origen. Y se trata también del relato de una decepción mayúscula al darse cuenta de que algunos proyectos de desarrollo, como el que quería implementar y etnografiar la autora, están mediados y controlados por ciertas Organizaciones No Gubernamentales para el Desarrollo (ONGD) y por líderes locales con intereses propios, que poco se relacionan con las necesidades de las personas. Sobre el enfoque teórico, Ana Toledo Chávarri enmarca esta obra, que es el resultado de su tesis doctoral, en la tradición de la antropología aplicada y, más concretamente, en la antropología para el desarrollo. Aunque se propone superarla, tengo la sensación de que su posición analítica es eminentemente postestructuralista, pues sigue bebiendo de las fuentes de Foucault y Escobar. Entiende el desarrollo, básicamente, como un discurso, un lenguaje que incorpora y reproduce una jerarquía o estructura de relaciones de poder. En otras palabras, el desarrollo como la creación de un dominio de pensamiento y acción. De igual forma, emplea los marcos de análisis de la economía política, la modernidad/ colonialidad y la teoría actor-red. Son estas primeras 200 páginas excesivamente teóricas, muy probablemente producto de la tesina del Diploma de Estudios Avanzados (DEA), y no contienen apenas información etnográfica de primera mano.

El principal aporte de esta monografía, en mi opinión, está en la segunda mitad del libro y no es otra que haber puesto el acento sobre el proceso de reconversión de una organización campesina en una ONGD. Como si de un plan de ajuste estructural se tratara, y a raíz de que el presidente Carlos Salinas de Gortari, el gran privatizador neoliberal, liquidara la Reforma Agraria, la Unión General Campesina, Obrera y Popular, A. C. (UGOCP) fue capaz de rearticularse física y discursivamente para mantener su existencia más allá de su razón de ser, abandonando la lucha por la tierra y demostrando un instinto de supervivencia corporativo al volcarse en la captación de recursos a través de los proyectos e instituciones de desarrollo. Era, por tanto, una organización campesina de inspiración zapatista relativamente joven -fundada en 1986- que, como tantas otras en México, surgieron al amparo de la Reforma Agraria revolucionaria. Pero en 1992, en lugar de plantar cara a la reforma del artículo 27 constitucional con que se ponía fin a su horizonte de sentido, fue capaz de colaborar con el Gobierno Federal a cambio de ciertas prebendas, obligándose a reinventarse -cuando lo coherente hubiera sido disolverse-, no sin pasar por una profunda crisis de identidad. Así, ha terminado por convertirse en una ONGD reconocida por instituciones internacionales de desarrollo como una organización con capacidades técnicas suficientes y experiencia en la ejecución de proyectos.

La UGOCP es una asociación civil de carácter nacional, con delegaciones en 19 de los 
32 estados mexicanos. Pero Toledo Chávarri centra su investigación en la región del Papaloapan, entre las entidades federativas de Oaxaca y Veracruz. Realiza un notable esfuerzo por contextualizar los momentos clave de la misma tanto en relación con la historia de México como con la antropología mexicana. En este vasto territorio, se hallan 357 municipios de población mayoritariamente mazateca y chinanteca, que se vieron profundamente afectados por la construcción de dos grandes presas hidráulicas. Estas intervenciones estatales se coordinaron desde la Comisión del Papaloapan, una institución pública semiautónoma, que perduró de 1946 a 1986, y donde confluyeron gran cantidad de actores, desde ingenieros y personal técnico a economistas y antropólogos, desde el mismísimo Juan Rulfo, que colaboró con el vocal ejecutivo Raúl Sandoval, hasta Mario Rodríguez Cruz, el hijo del entonces cacique regional mixe, Luis Rodríguez Jacob (Díaz Gómez, 1994: 194). Los miembros de la Comisión del Papaloapan y del Centro Coordinador Indigenista del Instituto Nacional Indigenista (INI) tuvieron que volar a Zacatepec para solicitar permiso oficioso a este cacique regional para la reubicación de casi 2.000 personas en el nuevo asentamiento de San Felipe Zihualtepec, municipio de San Juan Cotzocón, en la parte baja del Distrito Mixe (Luna Ruiz, 2003: 141-145).

En la construcción de la presa Miguel Alemán (1950-1954), se tuvieron en cuenta principalmente el control de las inundaciones y la producción de energía eléctrica. De los 22.000 desplazados, en su mayoría mazatecos, tan solo la mitad fueron reacomodados y muy pocos fueron los que recibieron indemnizaciones por sus tierras. Los responsables de dichos reacomodos fueron algunos destacados antropólogos mexicanos. "Las características ideológicas sobresalientes de los antropólogos adscritos a la Comisión del Papaloapan y al INI, que se reflejaban en el trabajo que realizaban, eran, a saber: un riguroso evolucionismo unilineal. Se consideraba que los mazatecos podían dar el gran salto evolucionista hasta la etapa civilizatoria, aprovechando la oportunidad de la reubicación en nuevos centros de población" (Nahmad Sitton y Zentella, 2010). Y aunque las buenas intensiones de estos colegas se dan por supuestas, lo que ocurrió en definitiva, en palabras de la autora, fue que "se primó el interés por lograr un desarrollo sobre el respeto a la diversidad cultural. La antropología se puso al servicio del Estado y del desarrollo" (página 108).

La construcción de la segunda presa, la del Cerro de Oro (1974 - 1990), se prolongó mucho más en el tiempo y halló mayor resistencia local. Terminó por unirse con la primera para formar una única masa de agua de más de $700 \mathrm{~km}^{2}$. Con un número similar de afectados, en este caso sobre todo chinantecos, el proceso de reubicación de la población y el papel de los antropólogos fue muy distinto. La antropología, por un lado, se había vuelto crítica y desencantada del ideal de progreso ilimitado. Además,

2. El mayor embalse español, y tercero europeo, es el de La Serena, en la provincia de Badajoz, con una extensión de unos $140 \mathrm{~km} 2$. 
el contexto económico internacional era muy diferente al de los años cincuenta y tuvo un impacto muy profundo en estas comunidades indígenas. De los petrodólares que habían inundado Latinoamérica -con los que se financió este segundo megaproyecto-, se pasó a la década perdida por arte de birlibirloque. De ser México la gran esperanza de modernización de un país del Tercer Mundo, se puso fin de forma irrevocable a toda posibilidad de industrialización por sustitución de importaciones. La crisis de la deuda de 1982, algo muy similar a lo que nos está ocurriendo en la actualidad a España, sirvió para materializar la dependencia exterior de la economía mexicana, desmontar el Estado asistencial y permitir a instituciones internacionales, como el Banco Mundial y el Fondo Monetario Internacional -ambas hijas de Bretton Woods-, dictar no ya las políticas económicas, sino también las sociales: educativas, sanitarias, de transporte, propiedad de la tierra, desarrollo, etc. En las comunidades, por ejemplo con el café y otras plantaciones, como con el Estado en su conjunto, con el petróleo, ocurrieron procesos muy similares. En un primer momento, se creó la dependencia externa de los mercados eliminando para ello estructuras productivas autosuficientes y vinculando tanto al Estado como a los campesinos mediante la deuda y los insumos externos. A continuación, la prolongada crisis económica acabó de doblegar las economías domésticas y nacionales al dictado del Imperio y sus transnacionales (Nash, 1994). En este contexto de retirada del Estado, se produce la emergencia de nuevos mediadores en las políticas de desarrollo, las ONG con su personal técnico, y de un nuevo paradigma del desarrollo: la participación. Ello, junto con la teoría del Capital Social del Banco Mundial y el llamado etnodesarrollo, llevará en los años noventa a una multiplicación todavía mayor de esos actores (organizaciones de base, ONGD de $2^{\circ}$ grado, indígenas, líderes locales, etc.) que son una parte central de la investigación de Toledo Chávarri.

El Proyecto de Mejoramiento Participativo de Plantas (MPP), al que le dedica por completo el capítulo VI, se centra en la implementación y el proceso de "textualización" de esta iniciativa en el Papaloapan, región donde se quería extender a partir de los buenos resultados conseguidos en Cuba. En primer término, el MPP surge como un proyecto de construcción de alternativas al modelo hegemónico neoliberal, concretamente a la Revolución Verde, mediante la participación de las poblaciones locales en la mejora de las semillas autóctonas. Se inició su ejecución en 2002, pero pronto se produjeron serios contratiempos (dos ciclones en el 2002 y un huracán en el 2005). En parte motivado por ello, cuando la antropóloga llega al campo, en 2006, el proyecto se ha visto reducido a meras presentaciones de PowerPoint y a algunos ficheros de Word. Además, los objetivos del mismo se han modificado, acorde al cambio que también está experimentando la propia UGOCP, pasando a ser la captación de financiación y el empleo de semillas híbridas, fines que incorporan y extienden la lógica del mercado y lo alejan de la concepción inicial. No en vano, el modelo que se pretende imitar es el de El Ejido, en Almería. Esta peculiaridad enfrentará a la UGOCP con otras ONGD que están implementando el MPP en otros 
países. Finalmente, durante los años 2006 y 2007, el proyecto en México solo perdurará en el papel, puesto que desaparece en la práctica. A pesar de ello, el MPP conseguirá revivir con una importante inyección de financiación de 238.000 dólares de la FAO, en el año 2008, lo que demuestra la desconexión de estos organismos internacionales con el campo y el contenido de los proyectos que financian -especialmente en su fase de implementación-. Ataques convulsivos que periódicamente les achacan a merced de la escena mediática internacional y la visualización de determinados problemas que se definen entonces como acuciantes: en este caso, la crisis alimentaria mundial de 2008, es decir, las hambrunas que se produjeron en algunos países africanos por la escasez de alimentos y el alza de los precios relacionada con la producción de agrocombustibles. Meros tratamientos, poco más que estéticos, de unos síntomas cuyas causas permanecen ocultas a los actores y redes involucrados en el desarrollo. Así, el proyecto se desliga hasta tal punto de los participantes a quienes estaba dirigido que en uno de los cuadros de esas presentaciones en que se ha convertido el MPP, donde se resaltan los logros y los retos, no aparecen los campesinos por ningún lugar (página 317). El objetivo real del proyecto es, a juicio de Toledo Chávarri, "reproducir la lógica clientelar de las redes internas y locales-regionales-nacionales de la UGOCP" (página 333). Es decir, lo que se pretende es tan solo reproducir el papel de mediadora de la ONGD, no solucionar o mejorar nada (Wolf, 1956: 1076), lo que pasa de forma necesaria por la captación de fondos para la organización -no ya para los proyectos o los beneficiarios-.

El resultado es la "Papaloanización" del MPP - como reconoce la misma autora, un concepto inspirado en la "Nepalización" (Pigg, 1992)-. Pero no en su definición. Porque, aunque se haya convertido ahora la participación en la nueva metáfora justificadora del desarrollo, la desconexión entre los planificadores y las necesidades locales siguió siendo grande. Por ello, los objetivos del proyecto no se pudieron negociar localmente. En cambio, durante la fase de implementación del MPP sí fue posible que los actores comunitarios se apropiaran del mismo para ajustarlo a la idiosincrasia local. Así, en la zona templada de la cuenca, el MPP ha servido para asegurar la estabilidad de los asentamientos y luchar contra la violencia. En cambio, en la zona fría, no se llevó a cabo, puesto que la UGOCP no consiguió las escuelas y carreteras en las que se había comprometido a mediar ${ }^{3}$.

Este último punto nos remite al tema de la mediación y el caciquismo. Si el interés por esta obra se basa en el segundo término de su título, los caciques, como era en gran medida mi caso, he de decir que no es uno de sus pilares teóricos. En realidad, la obra está atravesada por el uso popular-nativo del término "cacique", que no se define analíticamente, y, como tal, es del todo polisémico: aparecen caciques coloniales, terratenientes decimonónicos, acaparadores de la distribución, usureros, nuevos propietarios revolucionarios, líderes

3. Como queda patente en ambos casos, el mejoramiento de las plantas y la construcción de alternativas quedaron en un segundo plano. 
indígenas y ganaderos; llamados todos ellos “caciques”. También se apunta hacia los courtiers o los mediadores locales que implementan proyectos de desarrollo (Bierschenk y Olivier de Sardan, 1993). Son estos intermediarios los que extienden la lógica clientelar en el interior de las ONGD, pero no son considerados en la obra como caciques. El concepto de las redes clientelares se emplea con mayor precisión que el caciquismo, pero no se define la relación exacta que se da entre ambos términos.

Por otra parte, como aparece en la contraportada, son éstos "escenarios de neocolonialismo, corrupción política y violencia". No obstante, en mi opinión, se produce una excesiva normalización en el tratamiento de la corrupción. La cotidianidad de la misma en el contexto mexicano no debe ser un obstáculo para la capacidad de asombro y denuncia del antropólogo. En este sentido, se echa de menos una mayor rotundidad al describir fenómenos sociales abiertamente criminales y propios de una lógica política caciquil: nepotismo, desvío de fondos, amenazas, falta total de transparencia, distribución asimétrica de la masa salarial, expulsión de miembros después de relaciones "sexoafectivas", adulterio, poligamia, manifestaciones racistas hacia los propios afiliadosbeneficiarios ("chinantercos" y "mazatercos"), comentarios sexistas, etc. Sirva como ejemplo, el caso de un colaborador de la UGOCP que informa de que apenas cobra nada como salario, siendo su forma de conseguir recursos intermediar entre los grupos locales y los programas que gestiona la UGOCP -en un momento, llega incluso a amenazar con juntar 20 ejidos y ofrecérselos a otra organización-; se trata de una situación que la autora describe como la constatación de las relaciones de poder entre los líderes y los grupos de base, pero que más me parece una práctica de reproducción del caciciquismo a diferente escala (Iturralde Blanco, 2012: 43).

La UGOCP es una organización que se caracterizó, en su fase campesina, por el empleo de métodos violentos para conseguir arrebatar tierras a los terratenientes: ocupaciones de predios, bloqueos de carreteras e, incluso, sospechas de agresiones y homicidios. Llegó a ser denunciada por parte de Amanistía Internacional y ACNUR, pero Toledo Chávarri no profundiza en los motivos de las mismas. Desde la propia organización, un miembro describía esta violencia como lucha armada contras las "bandas blancas de los caciques". En estos mismos años, se produjeron también procesos de faccionalización dentro de las mismas comunidades entre partidarios de una y otra organización campesina, que luchaban también entre ellas por el reconocimiento de las mismas tierras (página 240). Finalmente, en un alarde de impunidad -tan habitual en México-, la propia UGOCP se vanagloria de su capacidad de manipulación de las elecciones. Algo tan característico de los sistemas de caciquismo, lo reproduce la propia UGOCP pidiendo a sus miembros que voten en las elecciones supramunicipales por el PRI, tanto en Veracruz como en Oaxaca. A decir de su dirigente, el mismo que fue asesinado junto a otros informantes en 2009, él es capaz de movilizar entre 75.000 y 90.000 votos solo en el estado de Veracruz. En los comicios municipales, intentan también controlar a las autoridades políticas 
presentando a sus candidatos bajo las siglas de hasta cuatro partidos políticos, lo que viene a demostrar que, en muchas comunidades, los emblemas de los partidos son poco más que etiquetas intercambiables.

Por último, la obra se completa con un prólogo de Salomón Nahmad Sittón, que hace las veces de introducción, y con un epílogo de Juan Carlos Gimeno, que es un apasianado alegato al compromiso político de los antropólogos y a no callarnos la enorme absurdidad que representa la violencia.

\section{REFERENCIAS BIBLIOGRÁFICAS}

Bierschenk, Thomas y Olivier De Sardan, Jean-Pierre (1993) "Les courtiers locaux du développement". Bulletin de l'APAD, 5, pp. 71-6.

Díaz Gómez, Floriberto (1994) "El caciquismo y la violación de los derechos humanos del pueblo mixe". En Salomón Nahmad Sitton (ed.) Fuentes etnológicas para el estudio de los pueblos ayuuk (mixes) del estado de Oaxaca. Oaxaca: CIESAS-Oaxaca / Instituto Oaxaqueño de las Culturas, pp. 553 - 570.

Iturralde Blanco, Ignacio (2012) "Autonomía comunitaria y caciquismo: identidad étnica, control social y violencia en una comunidad mixe de Oaxaca". ICIP - Working Papers 2012 - 2, pp. 7-57.

Luna Ruiz, X. G. (2003) De la cuenca a la selva. Política pública y reubicación en una comunidad indígena: San Felipe Zihualtepec, Oaxaca. México: Licenciatura Escuela Nacional de Antropología e Historia (ENAH).

Nahmad Sitton, Salomón y Zentella, Adriana (2010) “Introducción a los diarios de campo de Maurilio Muñoz en los estudios y reacomodos de la población mazateca y chinanteca de la presa Miguel Alemán de Temazcal, Oaxaca”. En Martín Felipe González Solano (ed.) Fuentes para la historia del indigenismo en México. Diarios de campo de Maurilio Muñoz en el Papaloapan 1956-1959. México, D. F.: Comisión Desarrollo de los Pueblos Indígenas - CDI, pp. 32-43.

Nash, June (1994) "Global Integration and Subsistence Insecurity". American Anthropologist 96, pp. 7 - 30.

Pigg, Stacy Leigh (1992) “InventingSocial Categoriesthrough Place:Social Representations and Development in Nepal”. Comparative Studies in Society and History 34, pp. 491-513.

Restrepo, I. (2009) “Crimen en Uxpanapa” en La Jornada 29/07/2009, Sección: Opinión. Wolf, Eric R. (1956) “Aspects of Group Relations in a Complex Society: Mexico". American Anthropologist 58, pp. 1065 - 1078. 